\title{
Nondestructive evaluation of oviposition behavior of the bamboo powderpost beetle, Dinoderus minutus, using X-ray computed tomography and acoustic emission
}

\author{
Hiroki Watanabe ${ }^{1 *}$ (D) Yoshiyuki Yanase ${ }^{2}$ and Yoshihisa Fujii ${ }^{2}$
}

\begin{abstract}
In this study, X-ray computed tomography (CT) and acoustic emission (AE) monitoring were used to nondestructively analyze the oviposition behavior of the bamboo powderpost beetle, Dinoderus minutus, which takes place inside bamboo culms. Newly mated adult females of $D$. minutus were singly exposed to a bamboo piece, obtained from culms of madake (Phyllostachys bambusoides), and were kept at a temperature of $28^{\circ} \mathrm{C}$ and a relative humidity of $65 \%$ with a photoperiod of $L 12: D 12$. The bamboo pieces were subjected to X-ray CT scanning every $2-5$ days, and they were also monitored continuously using AE. The behavior of males after mating was also analyzed in a similar manner. Oviposition tunnels bored across the bamboo vascular bundles and eggs deposited in the metaxylem vessels were visualized in the CT images. The females lived for $75 \pm 17$ days (mean \pm SD) after mating, and they created tunnels that were $116.4 \pm 52.1 \mathrm{~mm}$ in length and $177.2 \pm 78.9 \mathrm{~mm}^{3}$ in volume and laid $253.1 \pm 125.3$ eggs in total. Using AE monitoring, the tunneling activity of the females was detected. The AE data indicated that continuous tunneling activity of the females was separated by regular short pauses, lasting approximately $1 \mathrm{~h}$, occurring at intervals of around 2 days. The tunneling activity of the females did not exhibit clear circadian rhythms, suggesting that it was unaffected by external photoperiod. The males tunneled more slowly than the females and had no preference to the boring direction, and their activities were in synchronization with the photoperiod.
\end{abstract}

Keywords: Bamboo powderpost beetle, Dinoderus minutus, X-ray computed tomography, Acoustic emission, Oviposition

\section{Introduction}

Bamboo is an abundant natural resource that produces strong culms with high machinability and esthetic values. In Japan, bamboo culms have been used as construction and decorative materials in many traditional wooden houses, as well as for furniture and craft products. However, bamboo culms are susceptible to biodeterioration, even in seasoned conditions, and can be easily attacked by insects.

\footnotetext{
*Correspondence: watanabe-h@kyuhaku.jp

${ }^{1}$ Kyushu National Museum, National Institutes for Cultural Heritage, 4-7-2 Ishizuka, Dazaifu, Fukuoka 818-0118, Japan

Full list of author information is available at the end of the article
}

The bamboo powderpost beetle, Dinoderus minutus (Fabricius), (Coleoptera: Bostrichidae) is one of the most significant pests of dry bamboo culms in Japan [1-3]. Infestation of $D$. minutus begins by adults entering into and ovipositing inside bamboo culms in the manufacturing process, in storage, or in use. The hatched larvae bore tunnels to obtain nutrients, such as starch and sugar, from parenchyma tissue and develop into pupae. Adults also feed on bamboo after eclosion and leave via exit holes to mate [1-3]. In the processes of feeding and oviposition, bamboo tissues are turned into powder called frass, reducing the strength and esthetic qualities of the culms. 
Preventing the entrance and oviposition of beetles in undamaged bamboo culms is crucial for pest management. Early detection of beetle entrance and oviposition is also important because it may reduce the costs and effort necessary for extermination. Establishment of these control measures should be based on detailed information regarding oviposition biology; however, it is difficult to fully investigate the oviposition behavior of $D$. minutus because it is hidden inside the bamboo culms.

We previously demonstrated that X-ray computed tomography $(\mathrm{CT})[4,5]$ and acoustic emission (AE) monitoring $[6,7]$ were effective techniques for nondestructively analyzing the feeding and development of $D$. minutus. In this study, we applied these two techniques to analyze the oviposition behavior; X-ray CT was used to observe and quantify the extension of oviposition tunnels and the number of eggs laid by females, and $\mathrm{AE}$ monitoring was used to continuously record and analyze the tunneling activity of females after mating. For comparison, the behavior of mated males was also analyzed.

\section{Materials and methods}

\section{Preparation of experimental insects and bamboo} specimens

Pupae were collected from laboratory strains of $D$. minutus reared using culms of madake (Phyllostachys bambusoides). Because adults do not have apparent morphological sexual characteristics, sex identification was done during the pupal stage based on morphological differences between the last abdominal segments $[8,9]$. The pupae were then placed individually into a hole, 10 [longitudinal $(L)] \times 1.7$ (diameter) $\mathrm{mm}$, drilled in the end surface of a bamboo pieces, $30(L) \times 20$ [tangential $(T)$ ] $\mathrm{mm}$, for maturation. After closing the hole with a round bamboo rod, the bamboo pieces were kept separately in glass jars and were placed in an environmental chamber kept at a temperature of $28{ }^{\circ} \mathrm{C}$ and $65 \%$ relative humidity (RH). The bamboo pieces used throughout the series of experiments were prepared from of air-dry culms of $P$. bambusoides felled in May in Kyoto Prefecture, Japan.

After adult eclosion, the adult beetles remained and fed inside the bamboo pieces for approximately 10 days and then created exit holes on the bamboo surface. After the exit holes were observed, the adults were taken out and the pronota of males were marked with white ink to distinguish them from females. A total of 18 male and female pairs were prepared. Each pair was placed in a separate petri dish for 1 day. Mating was deemed to have occurred within this 1-day period, although only mating of four pairs was confirmed visually. The females were then placed individually inside a glass bottle with a new bamboo piece as an oviposition medium. Of the 18 females, 11 (F1-11) were singly exposed to single bamboo pieces [40 $(L) \times 40(T) \mathrm{mm}$ ] obtained from culm internodes, to which an AE sensor (R15 $\alpha$, Physical Acoustics Corp., USA) was attached with a cyanoacrylate adhesive (Fig. 1). They were subjected to both X-ray CT scanning and AE monitoring. Three other females (FX1-3) were also exposed individually to a bamboo piece of the same shape, but without an $\mathrm{AE}$ sensor, and were subjected only to X-ray CT scanning. In order to observe the oviposition behavior in more realistic conditions, two of the remaining four females (FL1 and FL2) were given larger bamboo internode pieces $[40(L) \times 140(T) \mathrm{mm}]$ and the other two (FN1 and FN2) bamboo pieces containing a node in the middle $[60(L) \times 40(T) \mathrm{mm}]$. The glass bottles containing a female beetle and a bamboo piece were returned to the environmental chamber kept at $28{ }^{\circ} \mathrm{C}$ and $65 \% \mathrm{RH}$, in which the average moisture content (MC) of the pieces was $11 \%$. In the environmental chamber, a photoperiod of L12:D12 (light period 6:00-18:00, dark period 18:006:00) was applied because the activities of adult beetles were estimated to be affected by outside light.

Bamboo pieces for individuals F1-11 and FX1-3 were replaced when the oviposition tunnel reached an edge of the pieces. When replacing a bamboo piece for individuals F1-11, the sensor was detached from the original piece and re-attached to the new piece.

To compare with females, nine male individuals (M19) after mating were subjected to X-ray CT scanning and $\mathrm{AE}$ monitoring. These males were exposed individually to a piece of bamboo internodes $[40(L) \times 30(T)$ $\mathrm{mm}$ ] with an AE sensor attached and were kept in the same environmental chamber in the same conditions as the females. The bamboo pieces for the males were not replaced.

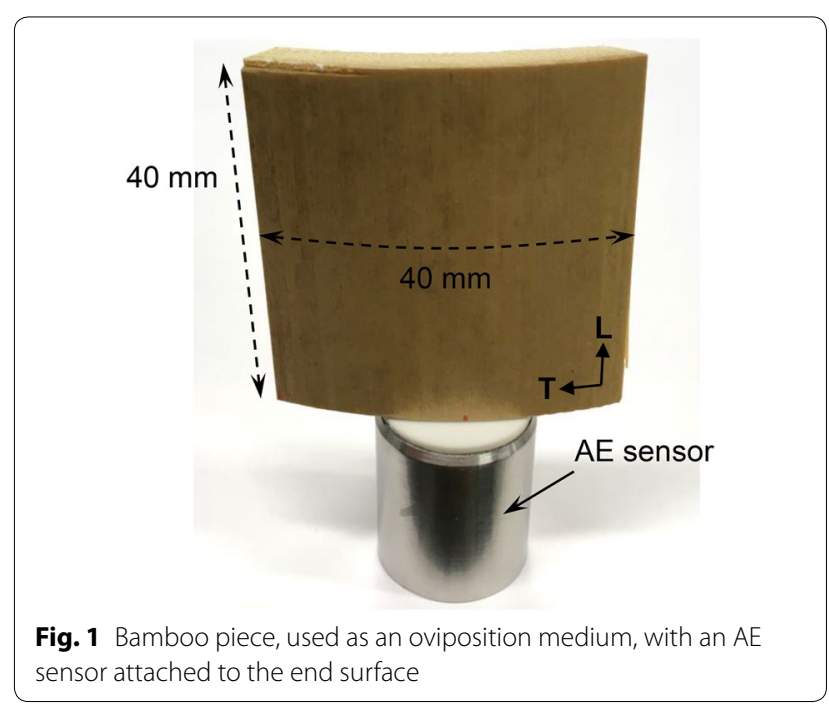




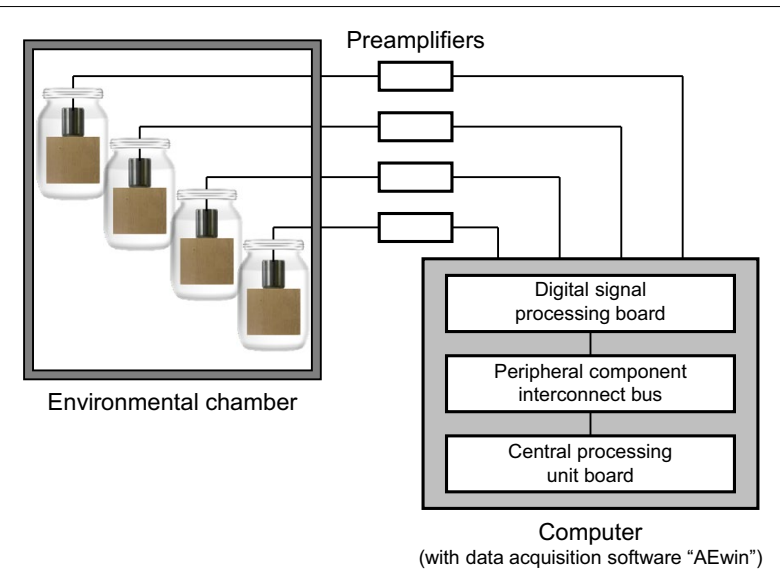

Fig. 2 Outline of the AE measurement system

\section{AE measurement (individuals F1-11, M1-9)}

The bamboo pieces were monitored continuously using $\mathrm{AE}$. The AE sensors used in this experiment had a resonant frequency of $150 \mathrm{kHz}$ and were connected to a 4-channel AE system (DiSP with AEwin, Physical Acoustics Corp., USA) via preamplifiers (1200A, Physical Acoustics Corp., USA) (Fig. 2). AE data acquisition in each channel was started as soon as the bamboo piece connected to the channel was placed together with one of the female individuals, F1-11, and the start was not necessarily set at a specific time of day for each individual. At most, four individuals were monitored at one time. $\mathrm{AE}$ signals detected by the sensors were filtered using $100-400 \mathrm{kHz}$ bandpass filter and amplified by $40 \mathrm{~dB}$ in the preamplifier and by $20 \mathrm{~dB}$ in the system amplifier. The signals were then discriminated at a threshold of $100 \mathrm{~dB}$, a level that would not be sensitive to low-amplitude signals generated by early instar larvae [7], and a signal that exceeded this threshold was recorded as an "AE hit".

The bamboo pieces with the males (M1-9) were monitored in the same manner, with the exception that AE monitoring was continued for only approximately 10 days.

The AE data were analyzed based on hourly AE hit rate, which represented the number of $\mathrm{AE}$ hits detected in $1 \mathrm{~h}$. To discuss the presence of circadian rhythms in the tunneling activity, a Chi-square periodogram analysis $[10,11]$ was applied to the time courses of hourly $\mathrm{AE}$ hit rate. In addition, another parameter, mean maximum amplitude (in $\mathrm{dB}$ ), was calculated as the hourly mean of six maximum amplitude values recorded during 10-min intervals when discussing the attenuation of $\mathrm{AE}$ waves in the bamboo culms.

To observe the frass-ejecting behavior and activities other than tunneling/feeding, the area around the tunnel entrance of four females (F1, F3, F5, and F11) and four males (M2, M3, M8, and M9) was observed using a webcam (UCAM-DLT30H, ELECOM Co., Ltd., Japan) for approximately 4 days. The images from the webcam were captured every 5 min.

An additional experiment was conducted to evaluate the effects of propagation distance and propagation direction of elastic waves on their attenuation, which seemed to appear prominently in the obtained AE data. Three bamboo specimens $[40(L) \times 70(T) \mathrm{mm}]$ were prepared from air-dry $(8 \% \mathrm{MC})$ culm internodes, which were $6-7 \mathrm{~mm}$ thick in the radial $(R)$ direction. Elastic waves were input from one end surface of each specimen, and the amplitude of the output waves was measured at the other end surface. Input signals of continuous sinusoidal waves with a frequency of $150 \mathrm{kHz}$ were generated using a digital function generator (DF1906, NF Corp., Japan) and were transduced and transmitted through a piezoelectric actuator (the same model as the AE sensors). A push pin was inserted into the end surface of the specimen as a waveguide. The transmitter was fixed to the head of the push pin, with silicone grease applied as an acoustic couplant between them. A receiver AE sensor was attached to the center of the other end surface of the specimen using a cyanoacrylate adhesive, and the output signals detected by the receiver were acquired using an AE tester (AE9501A, NF Corp., Japan). The amplitude of output signal waveforms displayed on a digital oscilloscope (DS1074Z, RIGOL Technologies Inc., China) was measured. The measurement system is summarized in Fig. 3 . The propagation distance and direction varied as

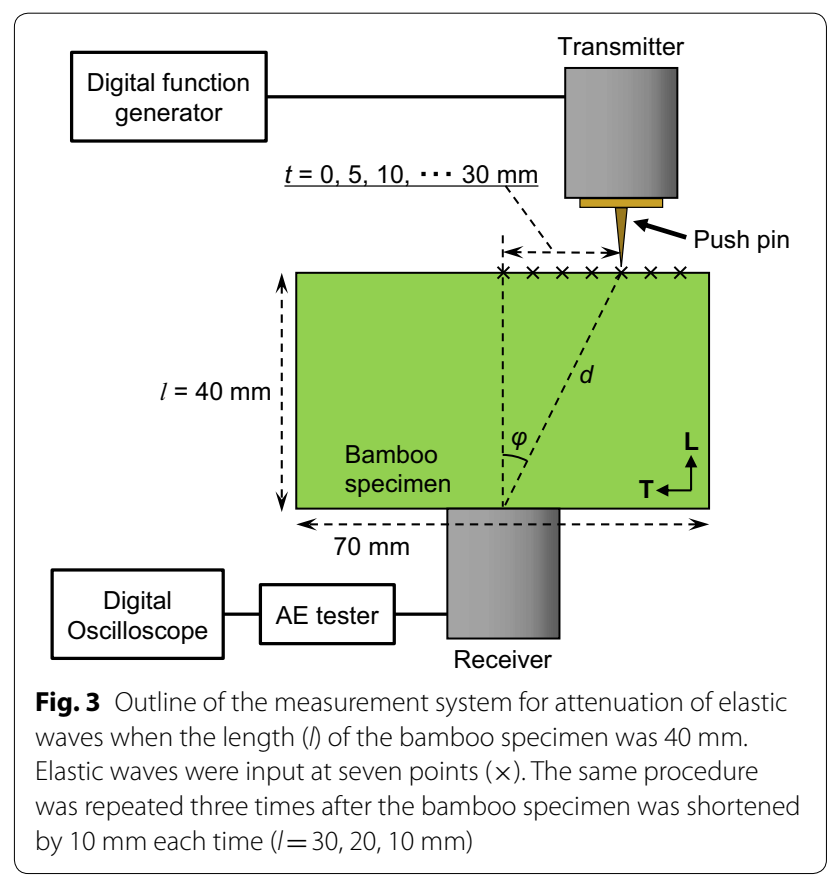


follows. Elastic waves were input at seven points where the tangential distance $(t)$ between the transmitter and receiver was increased in $5 \mathrm{~mm}$ steps from 0 to $30 \mathrm{~mm}$. Then, the specimen was shortened by $10 \mathrm{~mm}$ in the $L$ direction. The same procedure was repeated until the specimen's length $(l)$ was $10 \mathrm{~mm}$. Multiple linear regression was applied to correlate the distance between the transmitter and the receiver $(d)$ and the grain angle $(\phi)$, the angle between the $L$ direction and the line segment connecting the transmitter and the receiver, with the amplitude level of output signals $(A)$.

\section{X-ray CT scanning (all individuals)}

The bamboo pieces were scanned using a microfocus X-ray CT system (SMX-160CT-SV3S, Shimadzu Corp., Japan) at intervals of 2-5 days to visualize the tunnels and eggs. The X-ray source was operated with a tube voltage of $70 \mathrm{kV}$ and a tube current of $65 \mu \mathrm{A}$. The bamboo pieces for individuals F1-11 and FX1-3 were scanned with a resolution of $18 \mu \mathrm{m} / \mathrm{voxel}$, in which case the field of view was $9.2(x) \times 9.2(y) \times 8.7(z) \mathrm{mm}$. A different resolution, $60 \mu \mathrm{m} /$ voxel, was chosen when scanning the bamboo pieces for individuals FL1-2, FN1-2, and M1-9, in which case the field of view was $31(x) \times 31(y) \times 29(z) \mathrm{mm}$. The two scanning protocols were, respectively, referred to as "high magnification" and "low magnification" scans. For individuals F1-11 and M1-9, AE measurements were paused during the CT scans.

The CT images displayed using multi-planar reconstruction were used to measure the length and volume of the tunnels. For individuals F1-11 and FX1-3, the number of vascular bundles crossed and cut by oviposition tunnels and the number of eggs laid were also measured. Image processing software ImageJ (1.47v, W. S. Rasband, National Institutes of Health, USA) was employed for these procedures. The tunnel volume was estimated by assuming that the cross section of the tunnel was elliptical. The major and minor axes of the cross section of the tunnel were measured to calculate the cross-sectional area. The volume change from the previous scan was estimated by multiplying the cross-sectional area by the increase in tunnel length.

To discuss the relationship between oviposition and the pauses in tunneling activity, as observed by AE monitoring, individual F5 was subjected to daily CT scans for 7 days.

An additional experiment was conducted to measure the hatching rate of eggs, which could not be done using the CT images. Laminates of filter paper containing sugar and starch, as described by Watanabe et al. [5], were exposed to approximately 50 adults of unknown sex, and 50 randomly selected eggs laid between the laminates were collected and observed until they hatched or failed to. Egg collection and incubation were done in the same environmental chamber kept at $28{ }^{\circ} \mathrm{C}$ and $65 \% \mathrm{RH}$.

\section{Results and discussion \\ $X$-ray CT analysis of oviposition tunnels and number of eggs laid by females}

Individuals F8, F10, and FX1 were accidentally injured during handling, and individual F9 did not start oviposition. Therefore, the data from these four individuals were excluded from analyses, except where noted.

CT images of the bamboo pieces exposed to the mated females clearly captured the silhouettes of oviposition tunnels and eggs laid along the tunnels with a resolution of $18 \mu \mathrm{m} /$ voxel (high magnification). Figure 4 shows an example of merged CT images of individual F1 obtained 7 days after mating. In the CT images, hollow oviposition tunnels appeared darker and insect bodies, including adult beetles and eggs, appeared brighter. Internodes of bamboo culms mainly consist of vascular bundles, which are surrounded by sclerenchymatous fibers, and parenchyma ground tissue, and each vascular bundle has two metaxylem vessels $[12,13]$. In the CT images, dense parts like sclerenchymatous fibers also appeared brighter and the hollow inside of metaxylem vessels was darker. The females entered the bamboo pieces from the cross or R section, near the inner skin of bamboo, and bored across the vascular bundles in the $T$ direction, mostly along the inner skin. Because the females ejected frass from the entrance hole as they burrowed, the oviposition tunnels were hollow. Eggs were singly deposited inside the tubular metaxylem vessels that were cut open as the females bored. Not more than one egg was deposited in one open vessel. The shallower end of eggs was inserted $0.66 \pm 0.12 \mathrm{~mm}($ mean $\pm S D, n=15)$ deep below the cut end of the vessels. Additional direct dissection of one of the bamboo pieces after being replaced confirmed the presence of eggs as observed in CT images. It also indicated that the posterior ends were inserted first, with anterior ends facing the oviposition tunnels, and that the females plugged the vessels with a small amount of frass after inserting eggs. These patterns of egg-laying observed using X-ray CT agreed mostly with previous reports $[1-3,14-16]$.

Some newly hatched larvae were also captured in the CT images after an incubation period of approximately 5 days. They moved backwards, away from the oviposition tunnel, in the vessels after they hatched, and then entered the parenchyma. On average, they moved $14.4 \pm 8.8 \mathrm{~mm}$ (mean $\pm \mathrm{SD}, n=15)$ linearly in the vessels before entering into the parenchyma.

Figure 5 shows the time courses of the length and volume of oviposition tunnels and the number of eggs laid by individual F1, from mating to death. It entered 

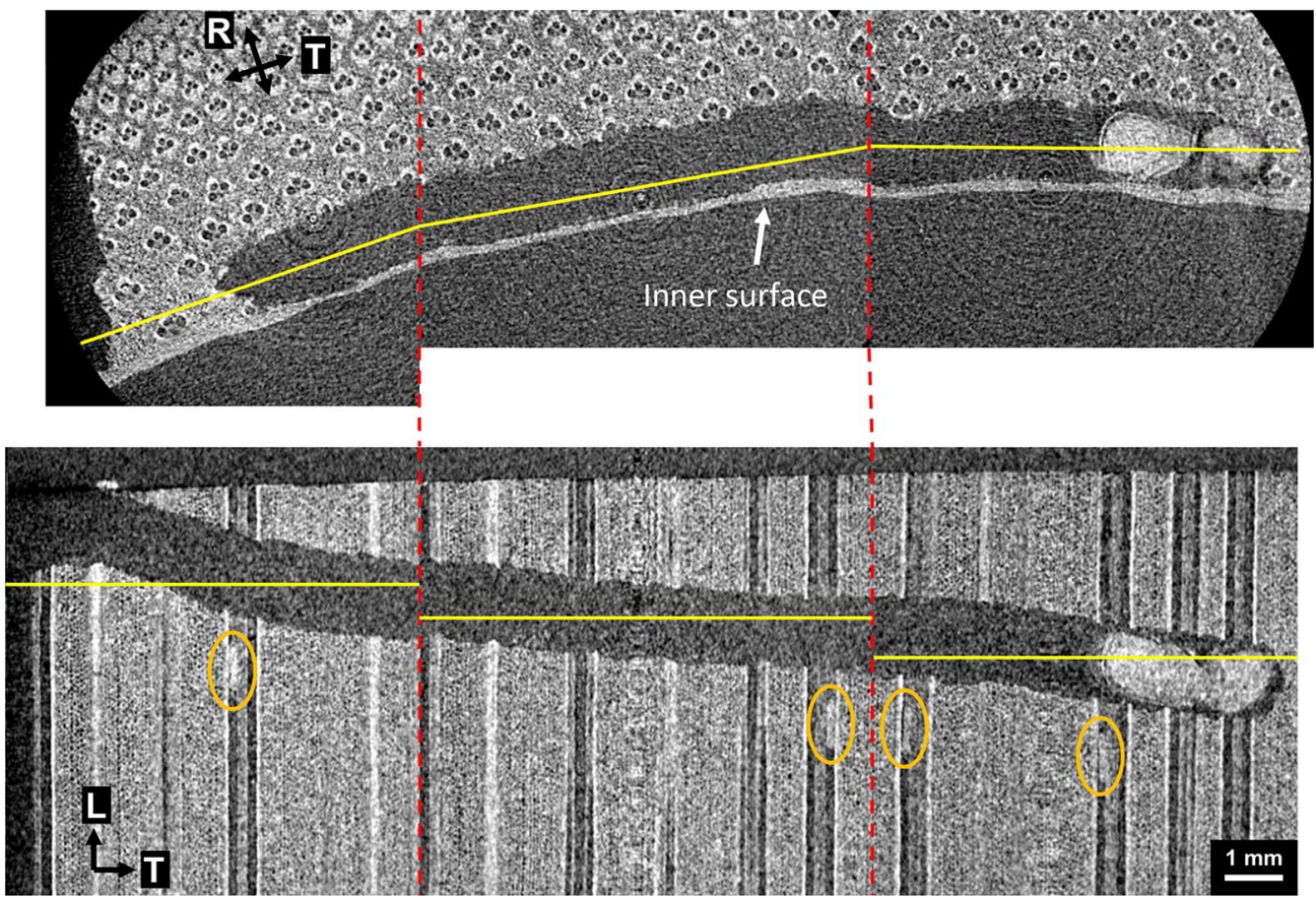

Fig. 4 Combined CT images capturing the oviposition tunnel of individual F1 and eggs (yellow circles) deposited in the metaxylem vessels, obtained using high magnification scans. Yellow lines indicate the positions of the corresponding planes

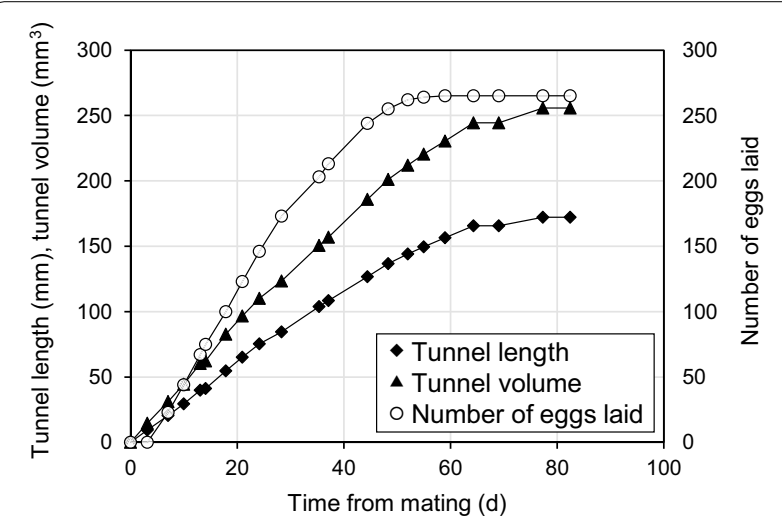

Fig. 5 Time courses of the length and volume of oviposition tunnels of individual F1 and the time course of the number of eggs laid

the bamboo piece soon after mating, and based on CT images, oviposition started 3 days after mating. The length and volume of the oviposition tunnel and the number of eggs laid increased linearly at first, but later the rates of increase declined. The last eggs laid were observed at 59 days, but the female continued tunneling until 77 days. It died at 82 days. The bamboo pieces were replaced three times; in total four pieces were used for this individual. The length and volume of oviposition tunnels reached $156.5 \mathrm{~mm}$ and $230.5 \mathrm{~mm}^{3}$,
Table 1 Periods of time from mating to the beginning of oviposition, from the beginning to the end of oviposition, and from the end of oviposition to death

\begin{tabular}{lllll}
\hline & $\begin{array}{l}\text { Mating-to- } \\
\text { oviposition } \\
\text { period }(\boldsymbol{d})\end{array}$ & $\begin{array}{l}\text { Oviposition } \\
\text { period (d) }\end{array}$ & $\begin{array}{l}\text { Post- } \\
\text { oviposition } \\
\text { period }(\boldsymbol{d})\end{array}$ & $\begin{array}{l}\text { Total post- } \\
\text { mating } \\
\text { lifespan } \\
(\boldsymbol{d})\end{array}$ \\
\hline Range & $0-7$ & $38-83$ & $2-32$ & $51-107$ \\
Mean $\pm S D$ & $2 \pm 3$ & $59 \pm 13$ & $15 \pm 10$ & $75 \pm 17$ \\
\hline
\end{tabular}

$S D$ standard deviation

respectively, by the end of oviposition, and a total of 265 eggs were laid. Until death, it created tunnels of $172.2 \mathrm{~mm}$ in length and $255.7 \mathrm{~mm}^{3}$ in volume in total. When the female crossed a bamboo vascular bundle, it cut two metaxylem vessel open, making four spots where eggs could be inserted. On average, 0.81 eggs were deposited in a single vascular bundle by individual F1.

Table 1 summarizes the durations of the mating-tooviposition, oviposition, and post-oviposition periods, and the total post-mating lifespan of 10 individuals, F1-7, F11, and FX2-3. The females started oviposition 0-7 (average 2) days after mating, and the oviposition period lasted 38-83 (average 59) days. They lived for 2-32 (average 15) days after the end of the oviposition 
period. Six of 10 individuals continued tunneling after the end of the oviposition period, whereas the other four stopped tunneling when the oviposition period ended.

Table 2 shows the tunnel size and the number of eggs laid by individuals F1-7, F11, and FX2-3. The females created tunnels of 31.4-209.8 (average 112.4) $\mathrm{mm}$ in length and 57.8-330.1 (average 171.1) $\mathrm{mm}^{3}$ in volume until the end of oviposition and laid 62-504 (average 253.1) eggs in total. A female laid 0.48-1.74 (average 1.23) eggs per vascular bundle. The final length and volume of the tunnels reached 32.3-214.1 (average 116.4) $\mathrm{mm}$ and $59.4-337.5$ (average 177.2 ) $\mathrm{mm}^{3}$, respectively. The hatching rate of eggs measured additionally using laminates of filter paper was $74 \%(n=50)$, suggesting that nearly three-quarters of eggs laid in bamboo culms should hatch into first instar larvae.

In addition, daily CT scans of individual F5 showed that this female laid eggs on a daily basis. This result is referred to in the next subsection to discuss the patterns of activity by the females.

In the larger bamboo pieces of $140(T) \mathrm{mm}$, individuals FL1 and FL2 normally extended a single oviposition tunnel in one direction. However, FL1 created three oviposition tunnels in total from different entrances, none of which reached an edge of the bamboo piece. The lengths of these tunnels were $36.9 \mathrm{~mm}, 63.4 \mathrm{~mm}$, and $13.2 \mathrm{~mm}$. FL2 reached the edge of the bamboo piece after tunneling for $89.6 \mathrm{~mm}$, and then made a new branched tunnel of $24.6 \mathrm{~mm}$ in a different direction. Individuals FN1 and FN2, given bamboo pieces containing a node, first entered from the $\mathrm{R}$ section at the node and created a rather "zigzag" oviposition tunnel roughly along the $T$ axis. The reason for this type of tunneling may be that some vascular bundles at bamboo nodes ran in $T$ and $R$ directions [12]. The length of tunnels made at the node was not measured because of their complex shape. The beetles moved to the internode to create a new tunnel after reaching the edge. Because eggs were not visualized in the CT images obtained using low magnification scans, the number of eggs was not counted for individuals FL1-2 and FN1-2.
Using X-ray CT, the spatial patterns of oviposition tunnels of $D$. minutus were assessed nondestructively, and the results were mostly consistent with previous reports. We also quantified the size of oviposition tunnels, i.e., the extent of damage caused by oviposition, which was not found in previous reports. The amount of damage by mated females is estimated to be more severe than that caused by growing larvae; $D$. minutus larvae consumed only $68.0 \mathrm{~mm}^{3}$ of bamboo on average within an average larval period of 61 days based on our previous study [5]. Furthermore, ovipositing females perpendicularly cut vascular bundles and sclerenchymatous fibers in bamboo culms, probably causing much more significant loss of culm strength compared with larvae, which usually tunneled in the fiber direction $[4,5]$.

This study also showed that X-ray CT was an effective tool to determine the fecundity of $D$. minutus. Previously, attempts were made to count the number of eggs laid per female using artificial diets [14, 17]. However, the number of eggs found in our study was much larger than in those reports. Plank [14] reported that 109.6 eggs were laid per female using corn as a diet. He also estimated that a female laid 119.4 eggs from mass rearing using bamboo. Norhisham et al. [17] used cassava pellets as an oviposition medium to investigate the effect of humidity on reproductive capacity of $D$. minutus. They examined the eggs laid inside the pellets daily and reported that 68.9 eggs were laid per female at a temperature of $30{ }^{\circ} \mathrm{C}$ and at an optimal $\mathrm{RH}$ of $85 \%$. Nondestructive analysis using X-ray CT may have reduced the effects of disturbance to the test beetles, allowing them to lay more eggs in a more natural environment than in previous studies, and it may also have improved the accuracy of observation. However, the difference in the results may also have been caused by other factors including experimental conditions and population differences of the test beetles.

The number of eggs laid by a female varied greatly among individuals (Table 2). As Fig. 6 shows, the body length, measured using CT images of the lateral view of the beetles, was significantly correlated with the number of eggs $(R=0.73, p<0.05)$. However, the body length of individuals F1-7, F11, and FX2-3 ranged only between 2.7 and $3.3 \mathrm{~mm}$, and it is uncertain what caused the

Table 2 Summary of length and volume of oviposition tunnels, number of vascular bundles cut, and number of eggs laid

\begin{tabular}{|c|c|c|c|c|c|c|c|}
\hline & \multicolumn{2}{|c|}{$\begin{array}{l}\text { Tunnels bored until the end } \\
\text { of oviposition }\end{array}$} & \multicolumn{2}{|c|}{ Tunnels bored until death } & \multirow{2}{*}{$\begin{array}{l}\text { Number of vascular bundles } \\
\text { cut during oviposition } \\
\text { period }^{\text {a }}\end{array}$} & \multirow{2}{*}{$\begin{array}{l}\text { Total } \\
\text { number } \\
\text { of eggs laid }\end{array}$} & \multirow[t]{2}{*}{$\begin{array}{l}\text { Number of eggs laid } \\
\text { per vascular bundle }\end{array}$} \\
\hline & Length (mm) & Volume $\left(\mathrm{mm}^{3}\right)$ & Length (mm) & Volume $\left(\mathrm{mm}^{3}\right)$ & & & \\
\hline Range & $31.4-209.8$ & $57.8-330.1$ & $32.3-214.1$ & $59.4-337.5$ & $61.5-404.5$ & $62-504$ & $0.48-1.74$ \\
\hline Mean \pm SD & $112.4 \pm 49.8$ & $171.1 \pm 75.6$ & $116.4 \pm 52.1$ & $177.2 \pm 78.9$ & $213.3 \pm 103.1$ & $253.1 \pm 125.3$ & $1.23 \pm 0.41$ \\
\hline
\end{tabular}

SD standard deviation

a One vascular bundle was deemed equivalent to two metaxylem vessels 


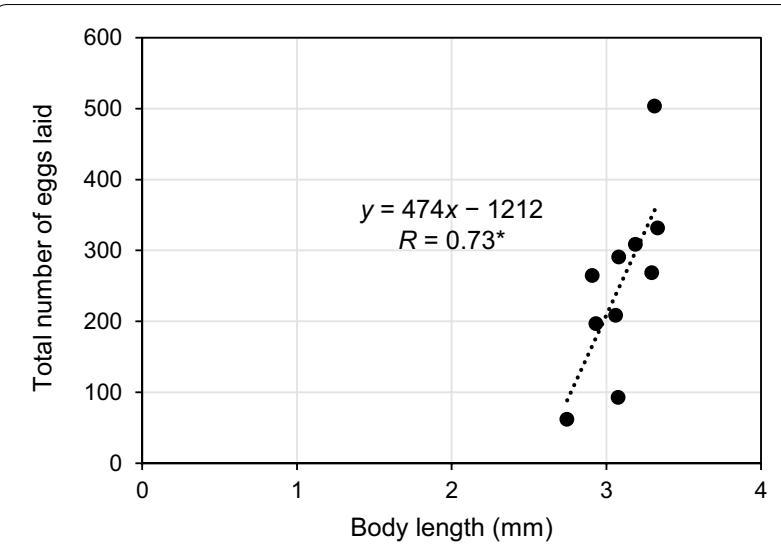

Fig. 6 Relationship between females' body length and total number of eggs laid. $\left(^{*}\right)$ correlation significant at 0.05 level

females to vary so greatly in fecundity. It should also be noted that, one individual, F9, did not oviposit, possibly because it had failed to copulate in the 1-day pairing period or had been congenitally infertile.

Based on microscopic and X-ray CT observations, an egg had a volume of approximately $0.015 \mathrm{~mm}^{3}$ and the volume of a female's abdomen was roughly measured to be $1.8 \mathrm{~mm}^{3}$. This meant that a female's abdomen only had capacity for approximately 120 mature eggs, whereas a female laid 253.1 eggs on average in its lifetime. This finding suggested that, as the females laid eggs, they took in nutrients and/or water from bamboo to develop immature eggs sequentially in the abdomen in order to produce eggs that, in total, exceeded the capacity of their own body. This may be another purpose for which $D$. minutus females continued tunneling during the oviposition period, besides making room for inserting eggs.

\section{AE monitoring for continuous analysis of tunneling activity \\ of females}

AE hits were continuously detected immediately after the bamboo pieces were exposed to the females. Based on our previous study on the relationship between the movement of the mandibles of $D$. minutus and the detection of $\mathrm{AE}$ waves [6], the generation of $\mathrm{AE}$ hits indicated that the females started biting the bamboo piece to bore into it.

Figure 7 shows the time course of the hourly AE hit rate of individual $\mathrm{F} 1$ as an example, for 60 days (a) and 10 days (b) after mating. To discuss the attenuation of AE waves, the time course of mean maximum amplitude of $\mathrm{AE}$ hits for the first 10 days is shown in Fig. 7c. Because the bamboo piece was replaced three times for F1, the graph in Fig. $7 \mathrm{a}$ is discontinuous at points where replacements occurred.
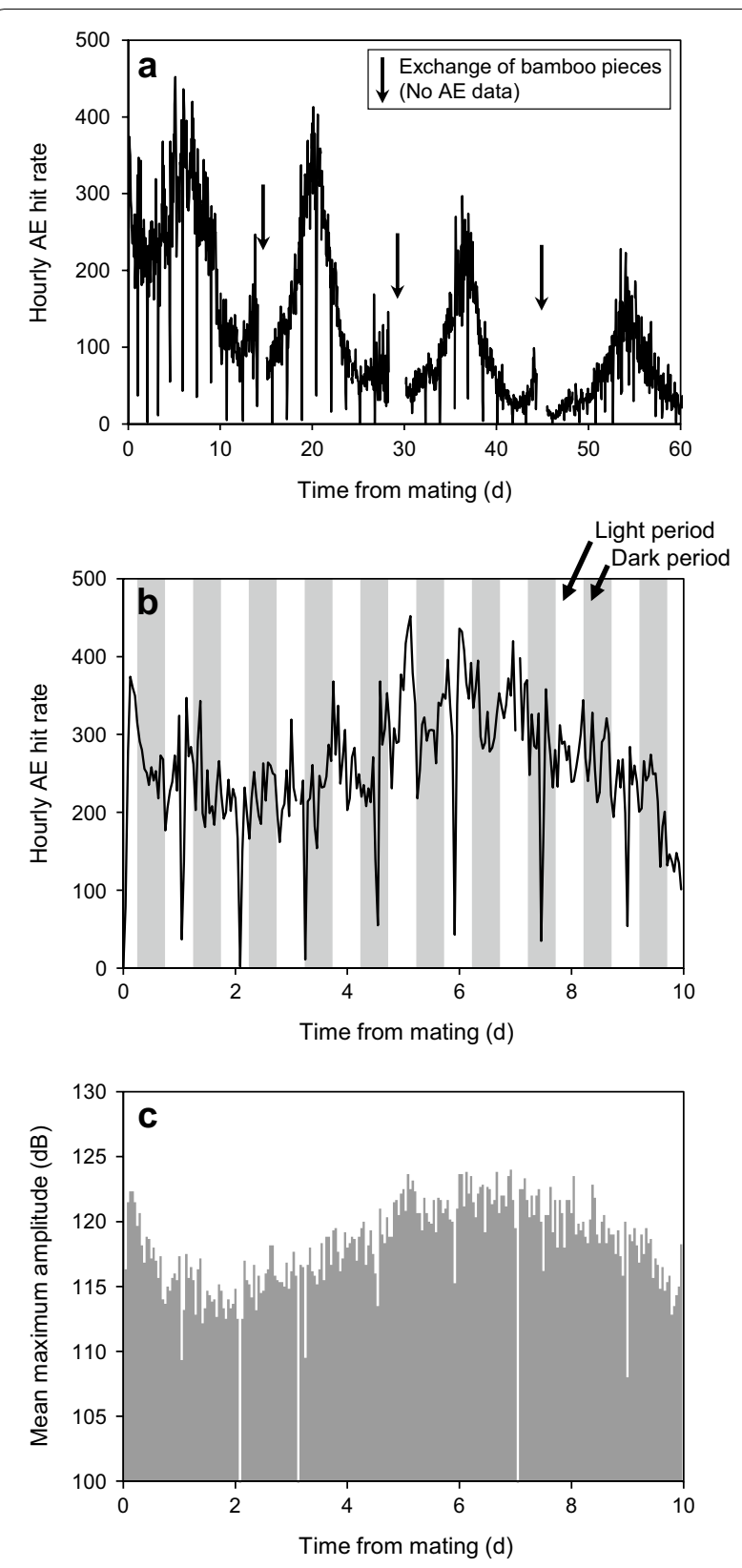

Fig. 7 Results of AE monitoring of individual F1. a Time course of the hourly AE hit rate for 60 days after mating. $\mathbf{b}$ Time course of the hourly AE hit rate for 10 days after mating, with light and dark periods indicated on the background in white and gray, respectively. c Time course of the mean maximum amplitude of AE for 10 days after mating

The graph of the hourly AE hit rate (Fig. 7a) seems to have two distinct patterns, one being a wave-like fluctuation with a period of approximately 15 days and one with short, temporary decrease occurring at intervals of 1-2 days, both of which were common in all analyzed females (F1-7, F11). Although the latter seems to 
originate from the beetles' activity, the former wavering pattern is probably unrelated to the beetles' activity and rather seems to be due to changes in the relative position of the beetle and the $\mathrm{AE}$ sensor. Because the $\mathrm{AE}$ hit rate (Fig. $7 \mathrm{~b}$ ) and mean maximum amplitude (Fig. 7c) showed almost the same tendency, the following assumption could be made. When the female entered the bamboo piece from one edge and tunneled in the $T$ direction, both the distance between the beetle and the sensor and the grain angle of the line between the beetle and the sensor decreased, both causing less attenuation of the $\mathrm{AE}$ waves and hence higher amplitude values of detected $\mathrm{AE}$ hits (e.g., at 6-7 days in Fig. 7c) and more AE hits (e.g., at 6-7 days in Fig. 7b). Then, after the beetle passed the midpoint and tunneled farther, contrasting decline in amplitude and AE hit rate occurred.

To verify this assumption, the effects of propagation distance and propagation direction of elastic waves in bamboo culms on attenuation were examined in an additional experiment. Figure 8 shows the results of this experiment, i.e., the amplitude levels of elastic waves detected by the receiver after propagating different distances at different grain angles (see Fig. 3). Multiple linear regression, with propagation distance $(d)$ and grain angle $(\phi)$ as explanatory variables and amplitude level $(A)$ as the dependent variable, showed that the effects of $d$ and $\phi$ on $A$ were significant $(A=83.35-0.40 d-0.24 \phi$; $R=0.89 ; p<0.001$ for $d$ and $\phi)$. This strongly suggested that the wave-like fluctuation in the time courses of $\mathrm{AE}$ amplitude and hourly AE hit rate was due to changes in the position of the females and thus did not reflect the females' activity.

The time course of the hourly $\mathrm{AE}$ hit rate in Fig. 7b showed that the female was almost continuously

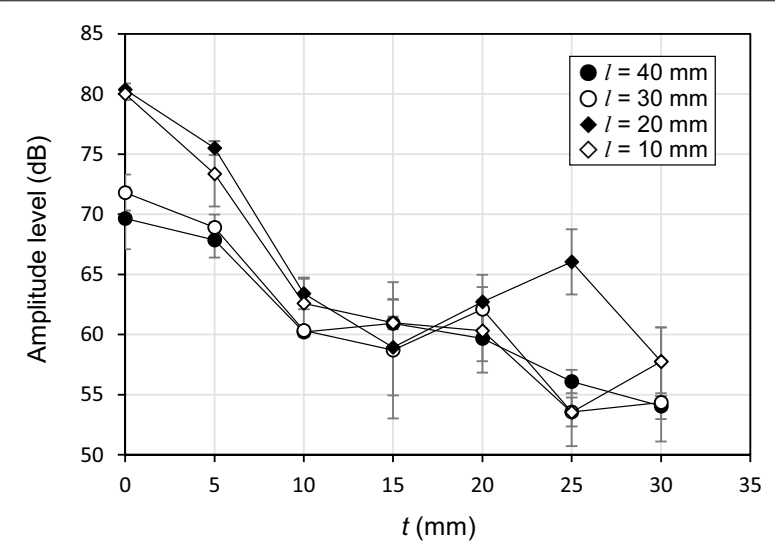

Fig. 8 Relationship between propagating distance $(t)$ in the $T$ direction and amplitude level of elastic waves for when the length (I) of the bamboo specimen was 40, 30, 20, and $10 \mathrm{~mm}$. Error bars standard deviation $(n=3)$ excavating an oviposition tunnel in both light (daytime) and dark phases (nighttime), but short pauses in tunneling activity, lasting approximately $1 \mathrm{~h}$, occurred at regular intervals. The intervals were longer than 1 day. Such a pattern was observed in all females (F1-7, F11), and the intervals of pauses were almost constant in one individual but differed among individuals, varying between 1.4 and 2.3 days. This pattern of females' tunneling activity seems to be independent of the $L 12: D 12$ photoperiod, the only possible zeitgeber (time-giver) in the experimental conditions, and no statistically significant circadian rhythms were present ( $p>0.05$; Chi-square periodogram [11]) in the time courses of the hourly AE hit rate of all females. This suggested that the tunneling activity of females, and hence oviposition, was not affected by outside light.

As will be shown later, the tunneling activity of mated males exhibited statistically significant circadian rhythms. Furthermore, our previous study showed that, in the Reifungsfrass period (the period of feeding by newly eclosed adults), the feeding activity continued almost ceaselessly, with no regular pauses, for approximately 10 days [7]. These findings suggested that the pattern in which short pauses in tunneling activity occur at certain intervals longer than 1 day is characteristic of the oviposition period of females.

We first assumed that the pauses in tunneling activity might correspond to either the moments of laying eggs or the moments of frass ejection. However, daily X-ray CT scans of individual F5 showed that oviposition occurred daily, even on days without the pauses, suggesting that eggs were deposited in the active periods, not in the pauses. The regular occurrence of pauses was also found in the infertile female, F9. These two findings suggested that the pauses in tunneling activity were not directly related to oviposition. Furthermore, direct observation of the tunnel entrance of individuals F1, F3, F5, and F11 using the webcam showed that they ejected frass in the active periods (4-9 times in a light phase), indicating that the pauses were not due to frass ejection. In addition, the internal events related to oviposition, such as fertilization of eggs, might occur during these pauses in tunneling activity. However, at this point, we were unable to further discuss this possibility without information regarding the morphological changes in the reproductive organs.

Females with longer intervals of pauses tended to lay eggs at greater rates. Using the data of individuals F1-7, F11, and the two individuals injured during the experiment, F8 and F10, there was a moderate correlation between the interval of pauses and the "initial rate of oviposition" ( $n=10, R=0.67$, significant at $p<0.05$ ) (Fig. 9). We defined the "initial rate of oviposition" as the number of eggs laid per day in the first 10 days, approximately, after oviposition started, and the interval of phases was 


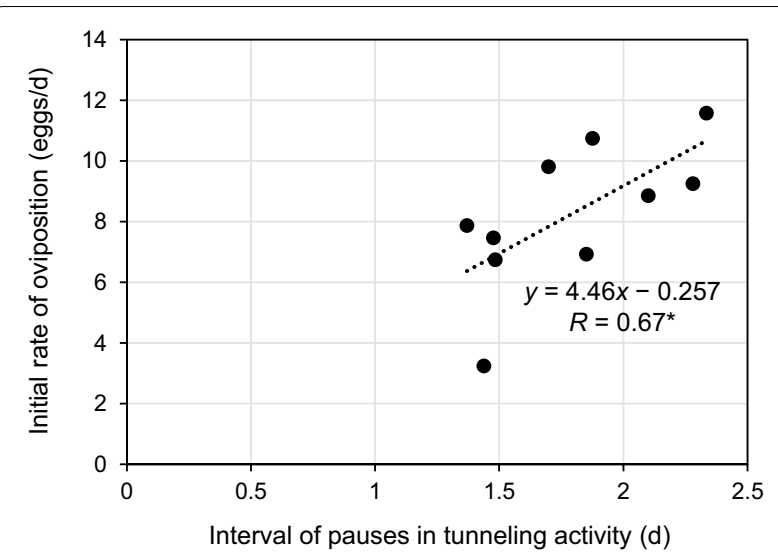

Fig. 9 Relationship between interval of pauses in the tunneling activity of females and initial oviposition rate. $\left(^{*}\right)$ correlation significant at 0.05 level

also measured in this 10-day period. From the X-ray CT data of individuals F1-7, F11, and FX2-3, there was a high correlation between the initial rate of oviposition and the total number of eggs laid in the lifetime $(n=10$, $R=0.82$, significant at $p<0.01$ ) (Fig. 10). Therefore, it was suggested that the length of the intervals of pauses may be related to the fecundity of females, although detailed investigations are needed to further discuss this phenomenon.

\section{Comparison of females' tunneling behavior to males'}

X-ray CT scans showed that the mated males (individuals M1-9) tunneled more slowly than the females and they had no apparent preference in their boring direction; the males created tunnels that were parallel, perpendicular, and oblique to the bamboo vascular bundles depending

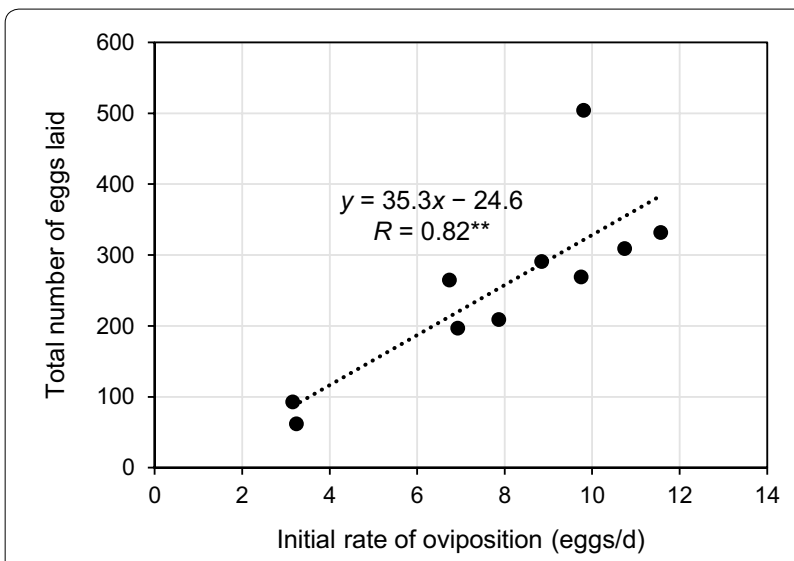

Fig. 10 Relationship between the initial rate of oviposition and total number of eggs laid. $\left(^{* *}\right)$ correlation significant at 0.01 level

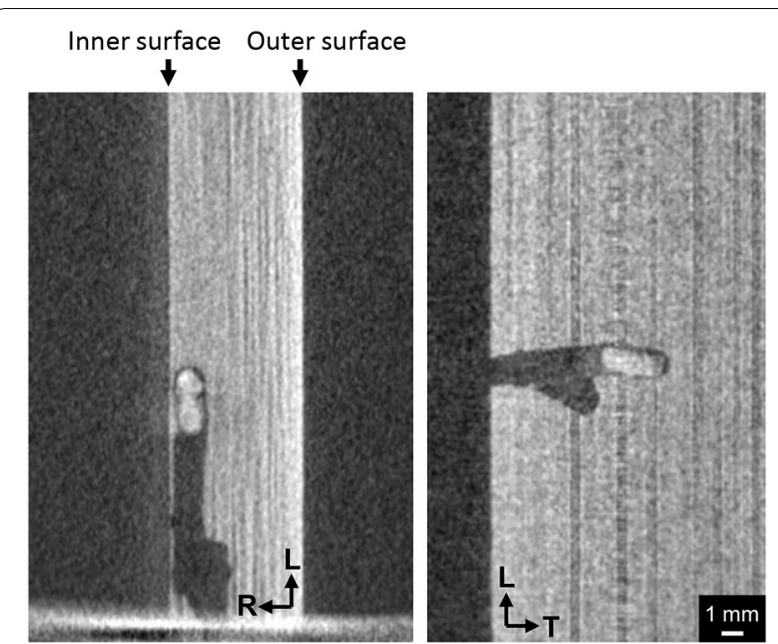

Fig. 11 CT images of tunnels of mated males, obtained using low magnification scans. Left tunnel of individual M1 bored longitudinally, right tunnel of individual M5, bored almost transversely to the fiber direction, with a branched oblique tunnel. Note that, on the left image, metal artifacts caused by the AE sensor are significantly visible at the bottom

on the individual. Figure 11 shows two examples of tunnels created by two male individuals, M1 and M5.

The males extended tunnels at average rates of $1.33 \pm 0.25 \mathrm{~mm} / \mathrm{d}$ in length and $2.22 \pm 0.56 \mathrm{~mm}^{3} / \mathrm{d}$ in volume $(n=9)$ in the first 10 days after mating, which were significantly smaller than those of the females $\left[2.73 \pm 0.75 \mathrm{~mm} / \mathrm{d}\right.$ and $4.25 \pm 1.26 \mathrm{~mm}^{3} / \mathrm{d}$, respectively $(n=10)](p<0.001$ for both; Welch's t test).

Because the bamboo pieces used for the males were smaller than those for the females, and because the rates of tunneling by the males were smaller, we assumed that the effects of attenuation of $\mathrm{AE}$ waves were less significant in the AE monitoring of the males than in that of the females.

The results of $\mathrm{AE}$ monitoring showed that the males had different temporal patterns of activity from the females, and their activities, unlike those of females, were dependent on outside light. Figure 12 shows the time course of the hourly AE hit rate of individual M1 for 10 days after mating. A statistically significant circadian rhythm was present in the tunneling activity of all males ( $p<0.001$; Chi-square periodogram [11]), and it was visually apparent that the tunneling activity of males was in synchronization with the photoperiod. The males started tunneling, or feeding, activity at 18:00, when the dark period started, every day. The exception was the first few days after mating, in case of individual M1, the first day. The tunneling activity continued almost ceaselessly in the dark periods and ended in the light periods, but unlike the start, the end of tunneling activity did not 


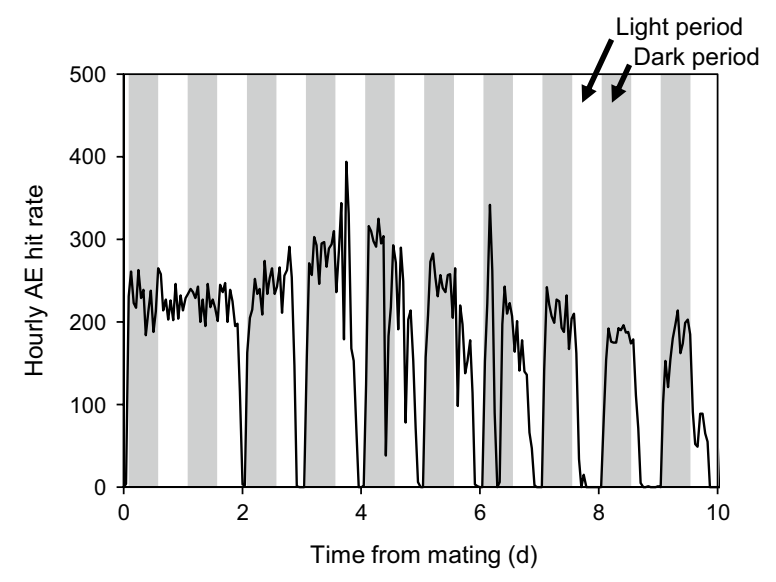

Fig. 12 Time course of the hourly AE hit rate of a male (individual M1). Light and dark periods indicated on the background in white and gray, respectively

strictly synchronize with the change in photoperiod, and the pause in tunneling activity tended to lengthen with elapsed days. Direct observation using the webcam suggested that the males were quiescent near the entrance of their tunnels during the pauses in tunneling activity. It was reported that $D$. minutus adults are most active outside when the light is fading [14], and the pauses in tunneling activity of males in the light periods may correspond to the periods in which they would exit to look for females in natural conditions. However, the reason why the males did not exit from the bamboo pieces in this experiment is unclear.

\section{Conclusions}

Using X-ray CT and AE monitoring, details of the oviposition behavior of $D$. minutus in bamboo culms were revealed nondestructively, without dissecting bamboo culms or using artificial diets, and the differences in behavioral patterns of post-mating females and males were also clarified. Based on the CT data, the females created tunnels that were $116.4 \pm 52.1 \mathrm{~mm}$ in length and $177.2 \pm 78.9 \mathrm{~mm}^{3}$ in volume from mating to death, along which they laid $253.1 \pm 125.3$ eggs in total. In contrast to oviposition tunnels that were mostly perpendicular to the fiber direction of bamboo, the tunnels of males were excavated in various directions depending on the individual, and the rate of tunnel extension was significantly lower than that of females. The results of AE monitoring indicated that mated females continued tunneling activity almost ceaselessly, but they regularly entered into a short state of inactivity at intervals of around 2 days independently of the photoperiod. This pattern of tunneling activity was estimated to be characteristic of the oviposition period. Males, on the other hand, exhibited activity patterns in synchronization with the photoperiod, and their tunneling (feeding) activity started with the beginning of the dark period and ended within the light period.

\begin{abstract}
Abbreviations
CT: Computed tomography; AE: Acoustic emission; L: Longitudinal; T: Tangential; RH: Relative humidity; MC: Moisture content; $R$ : Radial; $t$ : Tangential distance between the transmitter and the receiver; l: The length of the bamboo specimen; $d$ : The distance between the transmitter and the receiver; $\varphi$ : The angle (in degrees) between the $L$ direction and the line segment connecting the transmitter and the receiver; $A$ : The amplitude level of the output signals; SD: Standard deviation.
\end{abstract}

\section{Acknowledgements}

Part of this paper was presented at the 67th Annual Meeting of the Japan Wood Research Society (Fukuoka, Japan, 2017) and the 48th Annual Meeting of the International Research Group on Wood Protection (Ghent, Belgium, 2017).

\section{Authors' contributions}

All authors designed the research. HW conducted the experiments and data analyses and wrote the paper. All authors read and approved the final manuscript.

\section{Funding}

This work was supported in part by JSPS KAKENHI Grant Numbers JP19K13426 and JP17J04018.

\section{Availability of data and materials}

The datasets used and/or analyzed during the current study are available from the corresponding author on reasonable request.

\section{Competing interests}

The authors declare that they have no competing interests.

\section{Author details}

${ }^{1}$ Kyushu National Museum, National Institutes for Cultural Heritage, 4-7-2 Ishizuka, Dazaifu, Fukuoka 818-0118, Japan. ${ }^{2}$ Graduate School of Agriculture, Kyoto University, Kitashirakawaoiwake-cho, Sakyo-ku, Kyoto 606-8502, Japan.

Received: 13 March 2020 Accepted: 22 June 2020

Published online: 01 July 2020

\section{References}

1. Wood Technological Association of Japan (1961) Wood protection handbook. Shokodo, Tokyo (in Japanese)

2. Yamano K (1976) Insect pests of buildings. Sagami Shobo, Tokyo (in Japanese)

3. The Society of House and Household Pests Science, Japan (1995) Encyclopedia of house and household pests. Inoue Shoin, Tokyo (in Japanese)

4. Watanabe $\mathrm{H}$, Yanase $\mathrm{Y}$, Fujii Y (2015) Evaluation of larval growth process and bamboo consumption of the bamboo powder-post beetle Dinoderus minutus using X-ray computed tomography. J Wood Sci 61:171-177

5. Watanabe H, Yanase Y, Fujii Y (2017) Nondestructive evaluation of eggto-adult development and feeding behavior of the bamboo powderpost beetle Dinoderus minutus using X-ray computed tomography. J Wood Sci 63:506-513

6. Watanabe H, Yanase Y, Fujii Y (2016) Relationship between the movements of the mouthparts of the bamboo powder-post beetle Dinoderus minutus and the generation of acoustic emission. J Wood Sci 62:85-92

7. Watanabe $H$, Yanase Y, Fujii Y (2018) Continuous nondestructive monitoring of larval feeding activity and development of the bamboo powderpost beetle Dinoderus minutus using acoustic emission. J Wood Sci 64:138-148 
8. Aziz MA, Sitaraman NL (1948) Studies on the Dinoderus borer in bamboo: part II. identification of sexes in Dinoderus sp. Proc Indian Acad Sci 28:102-105

9. Abood F, Norhisham AR, Shahman M, Andy A (2010) Sexual identification of bamboo borer Dinoderus minutus (Fabricius) (Coleoptera: Bostrychidae). Malays For 73:1-6

10. Sokolove PG, Bushell WN (1978) The Chi square periodogram: its utility for analysis of circadian rhythms. J Theor Biol 72:131-160

11. Refinetti R. Circadian rhythm laboratory: periodogram. https://www.circa dian.org/periodogram.html. Accessed 26 Jan 2018

12. Sugiyama S (1983) Microscopic structure of bamboo culm-especially, on the arrangement of the vascular bundle in the bamboo stem of the Mosochiku (Phyllostachys pubescens MAZEL). Sci Bull Fac Educ Nagasaki Univ 34:89-109 (in Japanese)

13. Imai T, Majima S, Fujita M, Saiki H (1995) Cellular structures in culm internodes of three Phyllostachys species, Madake, Hachiku and Mosochiku (1). Bull Kyoto Univ For 67:147-157 (in Japanese)
14. Plank HK (1948) Biology of the bamboo powder-post beetle in Puerto Rico. Bulletin No. 44, Federal Experiment Station in Puerto Rico, Mayaguez

15. Garcia CM, Morrell JJ (2009) Development of the powderpost beetle (Coleoptera: Bostrichidae) at constant temperatures. Environ Entomol 38:478-483

16. Norhisham AR, Faizah A, Zaidon A (2015) Effects of moisture content on the bamboo borer Dinoderus minutus. J Trop Forest Sci 27:334-341

17. Norhisham AR, Abood F, Rita M, Hakeem KR (2013) Effect of humidity on egg hatchability and reproductive biology of the bamboo borer (Dinoderus minutus Fabricius). SpringerPlus 2:9

\section{Publisher's Note}

Springer Nature remains neutral with regard to jurisdictional claims in published maps and institutional affiliations.

\section{Submit your manuscript to a SpringerOpen ${ }^{\circ}$ journal and benefit from:}

- Convenient online submission

- Rigorous peer review

- Open access: articles freely available online

- High visibility within the field

- Retaining the copyright to your article

Submit your next manuscript at $\boldsymbol{\nabla}$ springeropen.com 